\title{
Transcriptome analysis reveals that TYRP1, linked with the Z-chromosome causes the green color of the feathers covering the head of male mallards through sex-biased expression
}

\author{
shengchao ma ${ }^{1}$, hehe liu ${ }^{2}$, jianmei wang ${ }^{3}$, lei wang ${ }^{1}$, yang $\mathrm{xi}^{2}$, yisi liu ${ }^{3}$, qian $\mathrm{xu}^{3}$, jiwei hu ${ }^{3}$, \\ chunchun han ${ }^{3}$, lili bai ${ }^{3}$, liang $\mathrm{li}^{3}$, and jiwen wang ${ }^{3}$ \\ ${ }^{1}$ Sichuan Agricultural University - Chengdu Campus \\ ${ }^{2}$ Affiliation not available \\ ${ }^{3}$ Sichuan Agricultural University
}

May 20, 2020

\begin{abstract}
Sexual dimorphism of plumage color is common in avians. A well-known example is mallard, in which drakes exhibit green head feathers, while females exhibit dull head feather color. Through microscopy observations, melanin was observed to be continuously deposited in feather barbules and to form a two-dimensional hexagonal lattice, which conferred the green feather coloration of drakes. Additionally, transcriptome analysis revealed that most pigmentation genes were highly expressed in feather follicles during the development of green feathers, which may contribute to melanin deposition. We identified 18 consensus differentially expressed genes in feather follicles by comparing the transcriptome differences in the male head vs. female head, male head vs. male back, and male head in the 7 th week vs. male head in the 11th week. Among these genes, TYRP1 located on Z-chromosome of the mallard genome, showed an increasing trend in the feather follicles of drake heads during green feather development. In particular, its expression was 256 and 32 times higher in the head follicles of males than in those of the female head and the male back, respectively. Hence, the green feathers were determined by TYRP1 through sex-biased expression, which is common for genes linked with Z-chromosome in avians. The differential expression of TYRP1 in different body parts of males and among different time points may be due to differences in cis-regulation by transcription factors. We also demonstrated that the beautiful feather color of other male avians is largely caused by the sex-biased expression of pigmentation genes linked with Z-chromosome.
\end{abstract}

\section{Key words}

Avian, Sexual dimorphism, Feather color, TYRP1 , Z- chromosome, Dosage effect

\section{Introduction}

Plumage color differences between males and females are common in most avian species, such as Anna's hummingbird, chicken, Japanese quail, mallard, turkey and zebra finch (Zuk et al. 1990, Zann 1996, Collins 1996). The nuptial plumage of adult males is beautiful, colorful and metallic, but the plumage color of females is dull. These characteristics are known as sexual dimorphism in avian species (Andersson 1994). Since Darwin, the function and evolution of sexual dimorphism in plumage color has been a popular field among evolutionary biologists (Darwin 1888). Some studies have revealed that sexually dichromatic species evolve from sexually monochromatic ancestors and that this trait elaboration is subsequently perfected by sexual selection (Andersson 1994). Avian species are more sensitive to colors than humans, as they can distinguish colors that humans cannot (Goldsmith 1991). Hence, plumage color is associated with the identification and courtship of avian species. The male's nuptial feathers give the carrier a mating advantage 
(Slagsvold \& Lifjeld 1992). Models of sexual selection have suggested that the feather color characteristics of males are flexible, reflecting the viability and body condition of the carrier (Zahavi 1975, Andersson 1986, Grafen 1990).

Mallard (Anas platyrhynchos) is one of the best models for studying feather color sexual dimorphism. The drake has metallic green feathers on its head, a white ring under its neck and brownish gray and stripes of feathers on the back, whereas the female duck has dark brown feathers on the head and back. The development of the metallic green (head) and blue (wings) coloration of mallards is very complex, as it is directly related to pigmentation deposition inside feathers. The pigments of organisms can be divided into two categories: carotenoids and melanins. Carotenoids can absorb light waves with wavelengths ranging from blue to green, which causes feathers to exhibit yellow, orange or red coloration (McGRAW \& Hill 2006). Melanins are more widely distributed and can be divided into eumelanin and phaeomelanin, which cause gray to black feathers and golden to rusty red feathers, respectively (Stavenga \& Wilts 2014). When these two kinds of melanin are mixed together, an intermediate color is produced (Hill 2010). Furthermore, these colors are related to coherent light scattering, which is caused by nanostructure inside the feathers (Prum 2006). This ordered nanostructure is formed by the assembly of melanosomes during feather development. This structure is analogous to a two-dimensional hexagonal lattice (Durrer 1997, Stavenga et al. 2017). The nuptial plumage color is neutral coloration in drakes, and the development of this neutral color is related to regulation by sex hormones (Witschi 1967, Ralph 1969). Estrogen inhibits the development of nuptial plumage in female ducks. The effect of testosterone is similar to that of estrogen in drakes (Haase et al . 1992). Sex hormones may affect the biosynthesis or transfer of melanin in cells.

Numerous functional genes determining feather color have been identified in avians through genetic studies. Most of these genes regulate the color of feathers by affecting melanocyte migration or melanin biosynthesis (Zhengkui Z et al , 2018, Li et al. 2012). Although studies have explained some of the causes of sexual dimorphism in plumage color in avian species (Johnsen et al. 2003, Hubbardet al. 2015, Hill 2000), its genetic basis is still unclear. Here, we used a duck model to investigate the genetic basis of green feather development in the drake head. First, we investigated the anatomical characteristics of the coloration of the head feathers and feather follicles of ducks. Then, genome-wide gene expression was analyzed by RNA-Seq, and genes related to green plumage color were screened. Our findings may provide a reference illustrating the mechanism of sexual dimorphism in the plumage color of avian species.

\section{Materials and methods}

\section{Animals and sampling}

The fertilized eggs of mallards were collected from the waterfowl breeding farm of Sichuan Agricultural University, Ya'an. After incubation, the hatched mallards were maintained for 14 weeks in a comfortable environment. These mallards could freely feed and drink throughout their growth stages. The head and back skin tissue of drakes and the head skin tissue of females were sampled at the ages of 7,11 and 14 weeks. Considering the difficulty of sampling feather follicles, we allowed the feather follicles to remain in the skin and sampled the skin tissue directly. Abundant feather follicles remained in each skin sample. In addition, feather samples were collected from 6- to 7-week-old and 14-week-old mallards. After sample collection was completed, some of the skin tissue samples were stored at $-80^{\circ} \mathrm{C}$, and the remaining skin tissue samples were stored in $4 \%$ paraformaldehyde, while the feather samples were stored in $3 \%$ glutaraldehyde.

\section{Light microscopy observations}

To investigate the anatomical structure of the skin, the skin samples were dehydrated with EthOH in successive concentrations of 70, 80, 90, 95, 99 and $100 \%$. The samples were further infiltrated three times in xylene. Each of these dehydration steps was performed in a dehydration system (Leica TM 1020, Germany) for $22 \mathrm{~h}$, and the tissues were then paraffin embedded. We investigated skin anatomy in cross-sections and vertical sections. The skin tissue samples were cut using microtome blades on a Leica RM 2135 microtome. The sections were stained with L-Dopa (Solarbio, China). Images of the tissue sections were obtained on an Olympus SZX16 stereomicroscope (Japan). Finally, the melanin content of the barb ridges was analyzed by 
using IPP 6.0 (Image-Pro Plus 6.0).

\section{Transmission electron microscopy observations}

Barbule anatomy was investigated by transmission electron microscopy (TEM) using standard methods (Thorpe et al. 2008). Briefly, a piece of feather was subjected to a series of dehydrations in acetone at increasing concentrations of $30,50,70,80,90,95$ and $100 \%$ and then infiltrated with epoxy resin at $3: 1,1: 1$ and 1:3 proportions. Next, we cured the resin blocks by heating them and cut them into approximately $50 \mathrm{~nm}$ sections using a Leica EM UC7 ultramicrotome (Leica Microsystems GmbH, Germany). The sections were stained using uranyl acetate and lead citrate. We checked the sections on a JEM-1400PLUS TEM (Japan). Finally, the number of melanosomes was analyzed by using IPP 6.0.

\section{RNA isolation and RNA-seq}

Total RNA was extracted from duck skin tissues using TRIzol (RNAiso Plus, Takara, Japan). Illumina platform sequencing of all samples was conducted at Novogene Bioinformatics Technology Co. Ltd, Beijing. In total, 15 sample libraries were constructed, including 12 libraries from head skin samples (three repeats from each stage of drake sampling in the $7^{\text {th }}, 11^{\text {th }}$ and $14^{\text {th }}$ weeks and three repeats from female mallards sampled in the $14^{\text {th }}$ week) and 3 from dorsum skin samples collected from drakes in the $14^{\text {th }}$ week. Low-quality reads (i.e., tags containing only adaptors and ambiguous bases) were removed from all samples.

\section{Bioinformatics analysis}

HISAT 2.0 software (Kim et al. 2015) ${ }^{[25]}$ was used to map the sequencing data with the assembly of the mallard reference genome (IASCAAS_PekingDuck_PBH1.5, GCF_003850225.1) to generate read count results. Second, the WGCNA (weighted correlation network analysis) software (Langfelder \& Steve 2008) of the $\mathrm{R}$ package was used to analyze the genome-wide gene coexpression pattern on the basis of all FPKM (fragments per kilobase per million) results. We used STEM (Short time-series expression miner) software to analyze the genome-wide expression trends in the partial FPKM results including three growth stages $\left(7^{\text {th }}\right.$ weeks, $11^{\text {th }}$ weeks and $14^{\text {th }}$ weeks) of drakes. Third, differential gene expression was tested by using DESeq 2.0 from the $\mathrm{R}$ package (Love 2014). The $\log _{2}$ (fold-change) values of the genes were tested by using DESeq to evaluate the expression patterns based on all sequencing data. The pathways of differentially expressed genes were analyzed by using clusterProfiler of the $\mathrm{R}$ package (Yu et al.2012). We used GraphPad 8.0, BioVenn and MeV 4.9 software to generate the volcano plots, Venn diagram and heat map of gene expression, respectively.

\section{Transcription factor prediction}

The online transcription factor (TF) binding site prediction tool (http://bioinfo.life.hust.edu.cn/AnimalTFDB/\#!/tfbs_predict) from the Animal TFBD database was used to predict the TF binding sites in the promoter regions of the TYR (Chr1- 8,264,483 82,694,830 bp) and TYRP1 genes (ChrZ- 307,517,598 30,752,598 bp).

\section{Synteny analysis}

In the synteny analysis, we analyzed six avian assemblies from NCBI (National Center for Biotechnology Information), including assemblies for mallard (IASCAAS_PekingDuck_PBH1.5), Anna's hummingbird (bCalAnn1_v1.p, GCF_003957555.1), chicken (GRCg6a, GCF_000002315.6), Japanese quail (Coturnix japonica 2.1, GCF_001577835.2), turkey (Turkey 5.1, GCF_000146605.3) and zebra finch (bTaeGut1_v1.p, GCF_003957565.1). These six species are representative avian species, and their genomes have been well annotated.

\section{Phylogenetic analysis}

We obtained the coding regions (CDs) of five genes (TYRP1 ,PTPRD , LURAP1L, MPDZ and NFIB ) of 6 avian species from the NCBI database (Table S1). We used Clustal Omega of MEGA 7.0 (Sudhir et al. 2016) to perform multisequence alignment of these sequences. Revised sequence alignments were submitted 
to MEGA 7.0 to construct a bootstrap (1000 replicate) tree (Felsenstein 1985) for each gene. The maximum likelihood (ML) method was used for phylogenetic analysis. With MEGA software, we identified the best DNA models for each gene (TYRP1 : K2+G, PTPRD : T92+G, LURAP1L : TN93+G,MPDZ : K2+I and NFIB : K2). The ML search started with the tree generated by BioNJ (Make initial tree automatically) (Gascuel 1997), and the optimal tree was determined via a heuristic search using the NNI (nearest-neighbor interchange) algorithm.

\section{Data statistics}

We performed all statistical analyses using $\mathrm{R}$ packages (Rteam et al. 2014). A p-value $<0.01$ was considered extremely significant, while a p-value $<0.05$ was considered statistically significant.

\section{Results}

\section{Anatomy of follicles and nanostructures of feather barbules}

The villi on the heads of the mallard ducklings began to transform into contour feathers at the age of 5 or 6 weeks. Initially, new feathers grew in areas near the eyes of drakes (Fig. 1B). At the $6^{\text {th }}$ to $14^{\text {th }}$ weeks, new feathers gradually grew in areas of the head and neck (Fig. 1B). At the $14^{\text {th }}$ week, the feathers finally turned green and covered the drakes' head entirely (Fig. 1B). Throughout the growth period of female mallards, the feathers in most areas were light in color (Fig. 1B) and did not change further.

To obtain a better understanding of green feather development, we performed histological observations. In the barb ridges of mallard drakes (Fig. 1D), melanin began to be deposited at the age of 7 weeks. At the ages of 11 and 14 weeks, the melanin content increased significantly (Fig. 1C and 1D). In female ducks, melanin deposition was not observed at the age of 14 weeks (Fig. 1C and 1D).

According to the observations of the vertical sections of the follicle pulp of males (Fig. 1D), melanin was mainly transferred from the dermal papilla to the barb ridges. At the age of 7 weeks, intense melanin deposition was observed in the follicle pulp. At the age of 14 weeks, less melanin was observed in follicle pulp areas. However, in females, there was still less melanin deposited in follicle pulp areas (Fig. 1D).

In the feathers, only the barbules at the top showed black coloration at the duck age of 5 weeks (Fig. $1 \mathrm{~F})$, which gradually changed from black to green at the ages of 6 to 14 weeks. We further investigated cross-sections of the small barbules between the black parts of feathers in the $6^{\text {th } \sim} 7^{\text {th }}$ weeks and the green parts of feathers in the $14^{\text {th }}$ week (Fig. $1 \mathrm{E}$ and $1 \mathrm{~F}$ ). In the barbules of the black parts, there were few melanosomes, and they were irregularly arranged under the keratin layer. In the barbules of the green parts, there were more melanosomes located under and enwrapped by the keratin layer, and they were arranged in an orderly pattern (4 layers) (Fig. $1 \mathrm{E}$ and $1 \mathrm{~F}$ ). Considering all of these results together, we suggest that the development of green feathers may be caused by the abundant melanin transferred to the small barbules. We further assumed that the excessive deposition of melanin/melanosomes caused the orderly arrangement of melanosomes and eventually led to green feather development through coherent emitted light scattering.

\section{Overview of RNA-Seq data}

A summary of the sequencing reads and matched genes is shown in Table S2. RNA-seq yielded an average of 3,140,000 raw reads from each RNA sample. After the low-quality reads were removed, an average of $3,000,000$ clean reads remained for each RNA sample. In addition, 18,024,093 reads on average were mapped to the reference genome for each RNA sample, with an average mapping rate of $93.2 \%$.

Genome-wide gene coexpression analysis of all 15 samples preliminarily reflected the relationships between gene expression patterns and phenotypes. The results showed that (Fig. 2) the cluster tree divided all samples into two categories: one contained most of the back samples, while the other contained most of the head samples (Fig. 2A). Then, the genes with similar expression patterns were divided into 11 modules (Fig. 2B). The correlation analysis between the modules and phenotypes showed that the gray module was correlated with the $14^{\text {th }}$ week head samples of females and males, while the brown module was correlated with the $14^{\text {th }}$ week head and back samples of males. We also found that the yellow module was correlated with all back and 
head samples from the $14^{\text {th }}$ week (Fig. 2D). In conclusion, the analysis of all of the RNA-Seq data effectively revealed the differences between samples. We found that the genome-wide gene expression patterns of the green and nongreen feather samples were different.

\section{Gene expression profile clusters during green feather development}

To understand the genes involved in the development of green feather color on the drake head, we performed gene expression profile cluster analysis based on the transcriptome data obtained at the time points of the $7^{\text {th }}$ week, $11^{\text {th }}$ week and $14^{\text {th }}$ week during green head feather development. The results showed that all genes could be divided into 16 categories (0-15), among which five significant categories, 14, 10,11, 15 and 9, were found. Among the five categories, the expression patterns of categories 14 and 10 and those of categories 11 and 15 were similar (Fig. 3A).

In these five significant categories, all genes were upregulated in the $7^{\text {th }}$ to $11^{\text {th }}$ weeks and downregulated in the $11^{\text {th }}$ to $14^{\text {th }}$ weeks. In category 10 , there were 16 genes, whose functions were related to melanocyte development (EDNRB , KIT , LYST ), melanin production (POMC , OCA2, TYR , TYRP1 ,TRPM1 , SLC45A2 , SLC24A4, SOX10, PAH ) and the melanin distribution (MYO5A, RAB27B , MLANA) (Cieslak et al. 2011, Jackson 1994, Bellone 2010, Sturm \& Duffy, 2012). Two other genes in category 9were related to melanin production $(M C 1 R, R A F 1$ ) (Cieslak et al. 2011, Jackson 1994, Bellone 2010, Sturm \& Duffy, 2012), while three genes in category 14 were related to melanin production (CLCN7, HPGDS, STX17 ) (Cieslak et al. 2011, Jackson 1994, Bellone 2010, Sturm \& Duffy, 2012). GO analysis further showed that (Fig. 3B and Table S3) signaling pathways associated with BMP and Wnt were significantly enriched. These signaling pathways were reported to play roles in melanin biosynthesis (Huang et al.2011) and feather development (Rouzankina et al. 2004). Hence, the transcriptomic analysis revealed that most pigmentation genes were highly expressed in feather follicles during the development of green head feathers, which may contribute to melanin deposition.

\section{The causative genes determining male green feathers}

To screen causative genes, we screened the differentially expressed genes (DEGs) among three comparisons: male head vs. female head, male head vs. male back, and male head in the $7^{\text {th }}$ week vs. male head in the $11^{\text {th }}$ week. In these comparisons, the results showed the enrichment of 1147, 297 and 177 DEGs, respectively were enriched in these comparisons. The GO analysis of the DEGs further showed that the TYR and TYRP1 genes were enriched in the pigment biosynthesis process, pigmentation, pigment metabolic, melanosome and pigment granule pathways (Tables S4, S5 and S6).

The potential causative genes responsible for the male green head feathers should explain the differences between all green and nongreen feather samples; thus, we checked the consensus DEGs among the three comparison pairs. Venn analysis identified 18 genes as potential causative genes determining the development of green male head feathers (Fig. 4B and. 4C). Among these genes, only TYR and TYRP1 were involved in melanin biosynthesis, which are key downstream genes of the three melanin synthesis pathways (cAMP, Wnt and MAPK pathways) (Fig. 4C), while others were related to receptor proteins, molecular binding and cellular structure. In the three comparison groups, the expression levels of TYR , SLC38A11 , REELD1 SYNPR ,LOC106019585, and TYRP1 were all upregulated, while only the LOC110352708 gene was downregulated in the three comparison groups (Fig. 4D). In the comparison of males vs. females, the expression of the TYRP1 gene was 256 and 32 times higher in the head follicles of males than in those of the female head and the male back, respectively. Among the 18 candidate genes, TYRP1 was located on the Z-chromosome, and the other genes (including TYR ) were located on euchromosomes.

Finally, because males have two Z-chromosomes while female mallards have only one, we compared the gene expression patterns of the head feather follicles of male and female mallards. We found that the expression levels of Z-chromosome-linked genes were higher in males than in females (Fig. 5A). Then, we analyzed the average $\log 2$ fold-change values of all genes on each chromosome, and we found that the Z-chromosome genes exhibited higher $\log 2$ fold-changes than those on all euchromosomes (Fig. 5B). Therefore, we believe that the double dosage effect of the Z-chromosome in males may lead to higher expression of the TYRP1 gene in 
male head feather follicles than in those of females.

\section{The cis-regulation of the $T Y R P 1$ gene may explain why only head feathers are green}

The transcription factor (TF) binding sites of the duck TYR and TYRP1 genes were predicted by using online prediction software with the promoters as input. All predicted TFs are provided in Tables S7 and S8. Binding sites of MAFA (MAF bZIP transcription factor A), GSC (goosecoid homeobox), OTX1 (orthodenticle homeobox 1) and FOXF2 (forkhead box F2) TFs were predicted in the TYR promoter region, while binding sites of MAFA, ARX (aristaless related homeobox) and FOXF2 were predited in the TYRP1 promoter region (Fig. 6A). Among these TF binding sites, $M A F A$ and $A R X$ binding sites were also found in 18 potential causative genes in the three comparison groups, while GSC, OTX1 and FOXF2 were differentially expressed only between the head follicles and back follicles of drakes (Table S9). Based on gene expression cluster analysis (Fig. 6B) of the drake samples, we observed that the expression patterns of $A R X$ and MAFA were most similar to those of the TYRP1 gene. The differential expression of TYRP1 between different body parts and time points in males was possibly due to differences in the cis-regulation of potential transcription factors.

\section{Pigmentation genes linked with the Z-chromosome may cause avian sexual dimorphism of plumage color}

Sexual dimorphism in plumage color is common in avian species. We further checked the pigmentation genes on the Z-chromosomes of six avian species showing sexual dimorphism of plumage color characteristics. The genomes of these six avian species were well assembled and annotated. Three pigmentation genes, MLANA , SLC45A2, and TYRP1, are located on the Z-chromosome (Table. S10). Synteny analysis (Fig. 7) showed that the TYRP1 gene and its neighboring genes were positioned according to their relative locations on the Z-chromosome. Four genes adjacent to TYRP1 , including PTPRD , LURAP1L, MPDZ and NFIB , were conserved in the six avian species and shared a similar transcription direction. Phylogenetic analysis (Fig. 7) further suggested that the above five genes presented similar phylogenetic relationships among the six avian species. These findings implied that the chromosomal segment carrying the TYRP1 gene and its neighboring genes was conserved in avian species, suggesting that the five other avian species may share a similar mechanism underlying sexual dimorphism in plumage color with mallards.

\section{Discussion}

The use of sexual dichromatism of plumage color as a model system in avian species helps us to understand sexual and natural selection and their roles in speciation. In avian species, females prefer bright plumage on males, which gives the more colorful males a mating advantage. In our work, we used mallards as a model to study the mechanism underlying the development of sexual dichromatism in plumage color.

As a characteristic of sexual dichromatism, the green feathers on the drake's head contrast sharply with the dull feathers on the female head. Previous studies have suggested that multiple mechanisms determine plumage color (Doucet 2009, Prum et al. 2009, Hill 2000, Maia et al. 2009, Maia). Among these mechanisms, pigmentation is important for feather coloration. Our anatomical observations showed that during drake development, melanin was continuously deposited in the head feather follicles. However, in the head feather follicles of females, melanin deposition was not observed at the age of 14 weeks. Haase et al . investigated the melanin contents of the feather follicles of male and female mallards by HPLC (high-performance liquid chromatography) and found that the content of eumelanin in the head feather follicles of drakes was significantly higher than that in the feather follicles of other body parts and the female head, which was consistent with our results ${ }^{[18]}$. After melanin was transferred from the feather follicles to the feather barbules on the drake head, the melanin/melanosomes were further arranged in an orderly pattern under the keratin layer of the feather barbules. Our results were comparable with the results of Doelele et al. (Doekele et al. 2017) and Eliason et al. (Eliason \& Shawkey 2012). Additionally, Eliasond et al. and Khudiyev et al. ( Khudiyev et al. 2014) found that this ordered arrangement was similar to a two-dimensional hexagonal lattice and caused the feathers to appear green by scattering light. The two-dimensional hexagonal lattice may be highly variable, allowing color to be altered by small variations in lattice constants, the melanosomal radius, 
and cortical thickness. Additionally, transcriptome data revealed that the expression of pigmentation genes began to increase during drake development and eventually remained high. These genes are mainly involved in the Wnt signaling pathway, MAPK signaling pathway (Concepción et al. 2001), melanosome formation and precursor transport. Collectively, these findings indicated that the development of green feathers was related to the excessive deposition and ordered arrangement of melanin in feather barbules.

We further revealed the underlying genes that determined the sexual dimorphism of mallard plumage color. First, we identified 18 consensus differentially expressed genes. Among these genes, only TYR and TYRP1 were involved in melanin biosynthesis. Tyrosinase (TYR) and tyrosinase-related protein 1 (TYRP1) regulate the development of 5,6-dihydroxyindoleic acid (DHICA), which is the precursor of eumelanin (Wang et al. 2017). Notably, the expression of the TYRP1 gene was significantly different in different body parts (head vs. back) and between the sexes (male head vs. female head). Therefore, we believe that the TYRP1 gene mainly determines the green feathers of males. Tobita-Teramoto et al. identified a 6-bp deletion that inactivated the TYRP1 gene in a line of albino chickens (Tobita-Teramoto et al. 2000). Xu et al . also showed that melanic plumage color in Korean quails may be associated with either increased production of $T Y R$ or decreased production of TYRP1 (Xu et al. 2013). All these studies also demonstrated the importance of the TYRP1 gene for feather color regulation.

Next, we studied the factors affecting the expression of the TYRP1 gene. We believe that the following two factors affect the expression of the TYRP1 gene: (1) the Z-chromosome dosage effect and (2) the cis-regulation of some TFs. Dosage compensation causes individuals with two or more copies of the gene in the nucleus to show the same phenotype as individuals with only one copy of the gene (Brockdorff \& Turner 2015). This phenomenon often occurs in mammals and insects. Unlike mammals and insects, when male avians acquired more sex chromosomes than females, the females of the avian species did not exhibit compensation. Thus, males tend to show expression higher levels of sex chromosome-linked genes than females (Wolf \& Bryk 2011, Mank 2013). Itoh et al. found that both zebra finch males and roosters exhibited up to $40 \%$ higher expression of Z-chromosome-linked genes than the females of these species (Itoh et al. 2007). Based on transcriptome analysis, we found that the Z-chromosome dosage effect also existed in mallards. Additionally, at least three pigmentation genes, including the TYRP1 gene, were located on the Z-chromosome of mallards and the other 5 other avian species. This suggests that the two TYRP1 genes may be expressed simultaneously in drake head feather follicles. The expression level of the TYRP1 gene in the head feather follicles of males was therefore higher than that in females. Finally, we found that the Z-chromosome segment containing the TYRP1 gene was conserved in six avian species. Based on the above results, we inferred that some pigmentation genes are linked to the Z-chromosome and may determine the sexual dimorphism of plumage color in avian species.

Eukaryotic transcription initiation is complex and requires the involvement of many transcription factors. In our work, we found that the expression of the TYRP1 gene was significantly lower in back feather follicles than in the head feather follicles of drakes. According to gene expression cluster analysis, the expression patterns of three transcription factors (MAFA, ARX and FOXF2) were similar to that of the TYRP1 gene, suggesting that the differential expression of TYRP1 among body parts (head vs. back) in drakes was mainly caused by transcription factors.

\section{Conclusion}

In the drake head, melanin/melanosomes are first deposited in feather follicles and then transferred to the barbules of green feathers. The melanosomes form a two-dimensional hexagonal lattice in barbules that causes feathers to emit green wavelengths of light. Most pigmentation genes were highly expressed during the above process. The TYRP1gene is located on the Z-chromosome and mainly determines the sexual dimorphism of mallard plumage color. The Z-chromosome dosage effect causes the expression of the TYRP1 gene in the male head to be higher than that in the female head. Additionally, the expression differences in the TYRP1 gene between the head and back of males are mainly regulated by some transcription factors. We thus assumed that the colorful plumage of male avians may be associated with the sex-biased expression of pigmentation genes linked to the Z-chromosome. 


\section{Additional files}

Additional file 1: Table S1. The genebank numbers of five genes in phylogenetic analysis.

Additional file 2: Table S2. RNA-Seq data summary and annotation results.

Additional file 3: Table S3. GO analysis of category 10 genes.

Additional file 4: Table S4. GO analysis of 11th week vs. 7th week.

Additional file 5: Table S5. GO analysis of head vs. back.

Additional file 6: Table S6. GO analysis of male vs female.

Additional file 7: Table S7. All transcription factors predicted results of TYR gene promoter.

Additional file 8: Table S8. All transcription factors predicted results of TYRP1 gene promoter.

Additional file 9: Transcription factors predicted results of TYRand TYRP1 promoters.

Additional file 10: Table S10. The common pigmentation genes linked with Z- chromosomes.

Data Accessibility

We plan to upload all transcriptome data to the SRA database of NCBI

\section{Acknowledgements}

Not Applicable

\section{Funding}

This work was supported by the National Natural Science Foundation of China (31872345), the Key Technology Support Program of Sichuan Province (2016NYZ0044), the China Agricultural Research System (CARS-43-6), and the National Key R\&D Program of China (2018YFD0501503-3).

\section{Author Contributions}

Firstly, Hehe Liu and Shengchao Ma conceived and designed the experiments. Secondly, Jianmei Wang, Yang Xi, Qian Xu, Jiwei Hu and Jiwen Wang and collected the materials, Shengchao Ma performed the experiments and analyzed the data. Lei Wang and Yisi Liu assisted complete the transcriptome analysis and paraffin sections, respectively. The manuscript was eventually written by Shengchao Ma and edited by all other authors. Finally, all authors endorse the final manuscript.

\section{References}

Andersson, M. (1994). Sexual selection. Princeton: Princeton University Press .

Andersson, M. (1986). Evolution of condition-dependent sex ornaments and mating preferences: sexual selection based on viability differences.Evolution , 40(4), 804-816.

Bellone, R. R. (2010). Pleiotropic effects of pigmentation genes in horses, Animal Genetics , 41, 100-110.

Brockdorff, N., \& Turner, B. M. (2015). Dosage compensation in mammals.Cold Spring Harb Perspect Biol , 7(3), a019406.

Collins, S. A., Cate, C. T. (1996). Does beak colour affect female preference in zebra finches?. Animal Behaviour , 52(1), 105-112.

Cieslak, M., Reissmann, M., Hofreiter, M., \& Ludwig A., (2011). Colors of domestication. Biological Reviews , 86(4), 885-899.

Darwin, C. (1888). The descent of man, and selection in relation to sex. London: John Murray . 
Olivares, C., Jiménez-Cervantes, C., Lozano, J. A., Solano, F., \& García-Borrón, J. C. (2001). The 5,6dihydroxyindole-carboxylic acid (DHICA) oxidase activity. Biochemical Journal, 354(Pt 1), 131-139.

Durrer, H. (1997). Schillerfarben der Vogelfeder als Evolutionsproblem.Denkschr Schweiz Naturforsch Ges , $91,1-126$.

Stavenga, D. G., van der Kooi, C. J, Wilts, B. D., (2017) Structural coloured feathers of mallards act by simple multilayer photonics. Journal of The Royal Society Interface , 14(133), 20170407.

Doucet, S. M. (2009). Structural Plumage Coloration, Male Body Size, and Condition in the Blue-Black Grassquit. The Condor, 104(Feb 2002), 30-38.

Eliason, C. M. \& Shawkey, M. D. (2012). A photonic heterostructure produces diverse iridescent colours in duck wing patches. $J$ R Soc Interface , 9(74), 2279-2289.

Felsenstein, J. (1985). CONFIDENCE LIMITS ON PHYLOGENIES: AN APPROACH USING THE BOOTSTRAP. Evolution, 39(4), 783-791.

Goldsmith, T. H. (1991). in The Perception of Color. In Vision and Visual Dysfunction (ed. Gouras, P.), Vol. 6, pp. 62-89. Macmillan, London

Grafen, A. (1990). Sexual selection unhandicapped by the Fisher process.J Theor Biol , 144(4), 473-516.

Gascuel, O. (1997). BIONJ: an improved version of the NJ algorithm based on a simple model of sequence data. Molecular Biology and Evolution , 14(7), 685-695.

McGRAW, K. J., \& Hill, G. E. (2006). Mechanics of carotenoid-based coloration. In Bird coloration, vol. 1 (eds Hill, G. E., McGraw, K. J.), pp. 177-242. Cambridge, MA: Harvard

University Press.

Haase, E., Ito, S., Sell, A., \& Wakamatsu, K. (1992). Melanin Concentrations in Feathers from Wild and Domestic Pigeons. Heredity, 83(1), 235.

Hubbard, J. K., Jenkins, B. R., Safran, R. J. (2015). Quantitative genetics of plumage color: lifetime effects of early nest environment on a colorful sexual signal. Ecology and Evolution ,16(5), 3436-3449.

Hill, G. E. (2000). Energetic constraints on expression of carotenoid-based plumage coloration. Journal of Avian Biology , 4, 559-566.

Hill, G. E. (2010). Bird coloration . Washington, DC: National Geographic.

Huang, Y. C., Yang, C. H., Chiou, Y. L. (2011). Citrus flavanone naringenin enhances melanogenesis through the activation of Wnt/ $\beta$-catenin signalling in mouse melanoma cells. Phytomedicine International Journal of Phytotherapy \& Phytopharmacology , 18(14), 1244-1249.

Itoh, Y., Melamed, E., Yang, X., Kampf, K., Wang, S., Yehya, N., .. Arnold, A. P. (2007). Dosage compensation is less effective in birds than in mammals. Journal of Biology , 6(1), 2.

Johnsen, A., Delhey, K., Andersson, S., Kempenaers, B. (2003). Plumage colour in nestling blue tits: sexual dichromatism, condition dependence and genetic effects. Proceedings of the Royal Society of London. Series B: Biological Sciences , 270(1521), 1263-1270.

Jackson, J. I. (1994). Molecular and Developmental Genetics of Mouse Coat Color. Annual Review of Genetics , 28(1), 189-217.

Kim, D., Langmead, B., \& Salzberg, S. L. (2015). HISAT: a fast spliced aligner with low memory requirements. Nature methods, 12(4), 357-360.

Khudiyev, T., Dogan, T., \& Bayindir, M. (2014). Biomimicry of multifunctional nanostructures in the neck feathers of mallard (Anas platyrhynchos L.) drakes. Scientific Reports , 22(4), 4718. 
Li, S., Wang, C., Yu, W., Zhao, S., \& Gong, Y. (2012). Identification of Genes Related to White and Black Plumage Formation by RNA-Seq from White and Black Feather Bulbs in Ducks. PLOS ONE , 7(5), e36592.

Langfelder, P., \& Steve, H. (2008). WGCNA: an R package for weighted correlation network analysis. BMC bioinformatics , 29(9), 559 .

Love, M. I., Huber, W., \& Anders, S. (2014). Moderated estimation of fold change and dispersion for RNA-seq data with DESeq2. Genome Biology , 15(12), 550.

Maia, R., Caetano, J. V., Báo, S. N., \& Macedo, R. H. (2009) Iridescent structural colour production in male blue-black grassquit feather barbules: the role of keratin and melanin. Journal of the Royal Society Interface , 6(Suppl 2), S203-S211.

Maia, R., Brasileiro, L., Lacava, R. V., \& Macedo R. H. (2012) Social environment affects acquisition and color of structural nuptial plumage in a sexually dimorphic tropical passerine. PloS one ,7(10), e47501.

Mank, J. E. (2013). Sex chromosome dosage compensation: definitely not for everyone. Trends in genetics , 29(12), 677-683.

Prum, R. O. (2006). Anatomy, physics and evolution of structural colors. In Bird coloration, vol. 1 (eds Hill, G. E., McGraw, K. J.), pp. 243-294. Cambridge, MA: Harvard University Press.

Prum, R. O., Andersson, S., \& Torres, R. H. (2009). Coherent Scattering of Ultraviolet Light by Avian Feather Barbs, The Auk, 120(Jan 2003), 163-170.

Ralph, C. L. (1969). The control of color in birds. American Zoologist, 9(2), 521-530.

Slagsvold, T., \& Lifjeld, J. T. (1992). Plumage color is a condition-dependent sexual trait in male pied flycatchers.Evolution, 46(3), 825-828.

Rteam R, Team RDC, Team R, et al. (2014) R: a language and environment for statistical computing, Computing, 14, 12-21.

Rouzankina, I., Abate-Shen, C., Niswander, L. (2004). Dlx genes integrate positive and negative signals during feather bud development.Developmental Biology , 265(1), 219-233.

Stavenga, D. G., Wilts, B. D. (2014). Oil droplets of bird eyes: microlenses acting as spectral filters. Philosophical Transactions of the Royal Society B: Biological Sciences , 369(1636), 20130041.

Sudhir, K., Glen, S., \& Koichiro, T. (2016). MEGA7: Molecular Evolutionary Genetics Analysis Version 7.0 for Bigger Datasets. Molecular Biology \& Evolution , 33(7), 1870-1874.

Sturm, R. A., \& Duffy, D. L. (2012). Human pigmentation genes under environmental selection. Genome biology , 13(9), 248.

Thorpe, J. R., Tang, H., Atherton, J., \& Cairns, N. J. (2008). Fine structural analysis of the neuronal inclusions of frontotemporal lobar degeneration with TDP-43 proteinopathy. Journal of Neural Transmission, 115(12), 1661-1671.

Tobita-Teramoto, T., Jang, G. Y., Kino, K., \& Salter, D. W., Brumbaugh, J.,\& Akiyama, T. (2000) Autosomal albino chicken mutation (c(a)/c(a)) deletes hexanucleotide (-Delta GACTGG817) at a copper-binding site of the tyrosinase gene, Poultry Science, 79(1), 46-50.

Witschi, E. (1961). Sex and secondary sexual characters. Biology and comparative physiology of birds 2 (Ed. Marshall, A. J.).

Wang, Y., Viennet, C., Robin, S., Berthon, J. Y., He, L., \& Humbert, P. (2017) Precise role of dermal fibroblasts on melanocyte pigmentation. Journal of Dermatological Science , 2017, 88(2), 159-166.

Wolf, J. B. W., \& Bryk, J. (2011). General lack of global dosage compensation in ZZ/ZW systems? Broadening the perspective with RNA-seq.BMC genomics , 12(1), 91 . 
Xu, Y., Zhang, X. H., \& Pang, Y. Z. (2013). Association of tyrosinase (TYR) and Tyrosinase-related Protein 1 (TYRP1) with melanic plumage color in Korean quails (Coturnix coturnix).Asian-Australasian journal of animal sciences , 26(11), 1518-1522.

Yu, G., Wang, L. G., Han, Y., \& He, Q. Y. (2012). clusterProfiler: an R Package for Comparing Biological Themes Among Gene Clusters. Omics A Journal of Integrative Biology , 16(5), 284-287.

Zuk, M., Thornhill, R., Ligon, J. D., \& Johnson, K. (1990) Parasites and mate choice in red jungle fowl. American Zoologist, 30(2), 235-244.

Zann, R. A. (1996). The zebra finch: a synthesis of field and laboratory studies. Oxford: Oxford University Press.

Zahavi, A. (1975). Mate selection - a selection for a handicap. Journal of theoretical Biology , 53(1), 205-214.

Zhou, Z., Li, M., Cheng, H., Fan, W., Yuan, Z., Gao, Q., .. Jiang, Y. (2018). An intercross population study reveals genes associated with body size and plumage color in ducks. Nature Communications , 9(1), 2648-2657.

Figures 

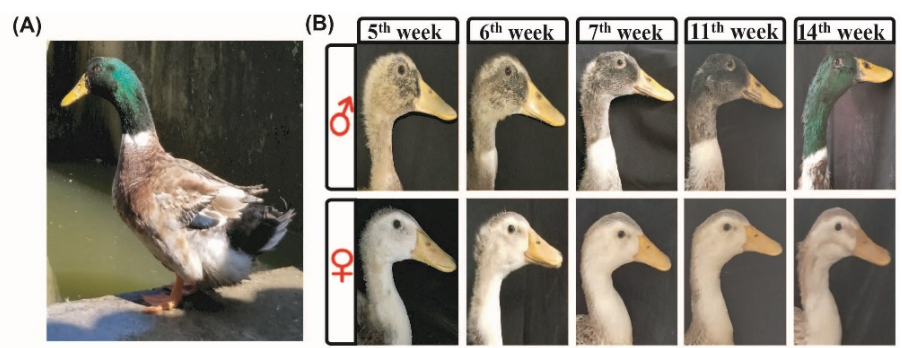

(C)
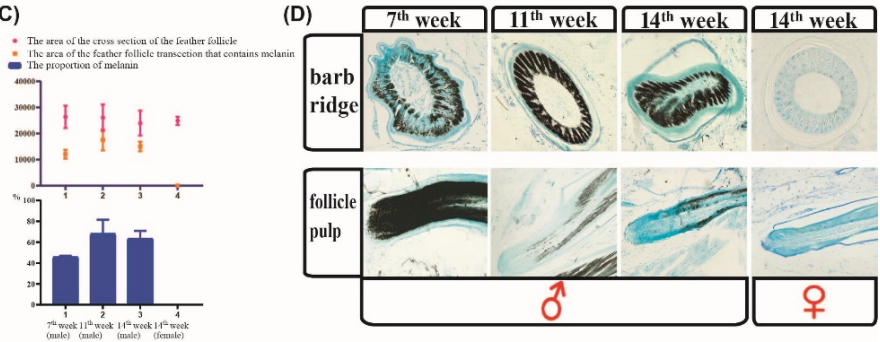

(D)

(E)

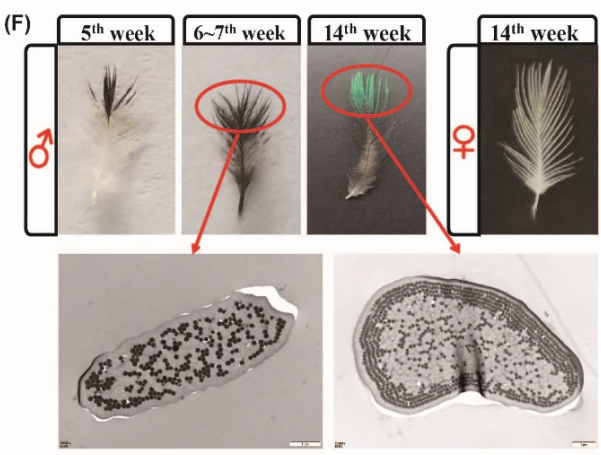

Figure 1. The difference in feather color between males and females revealed by microscopic and anatomical observations. (A) Image of a male mallard. (B) Color changes of the head feathers in mallards at different growth stages. (C) The melanin content in the barb ridges of mallards was measured by using IPP software. (D) Cross-sections of the barb ridges and vertical sections of the feather follicle pulp in mallards at different growth stages. (E) The number of melanosomes in the barbules of mallards was calculated by using IPP software. (F) The feathers were isolated from the head of male and female mallards at different growth stages. TEM (transmission electron microscope) images of vertical sections of the small barbules; scale bar $=1 \mu \mathrm{m}$. 
(A)

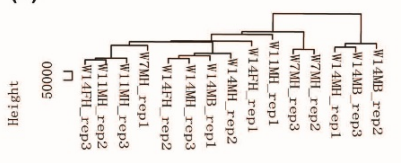

(C)

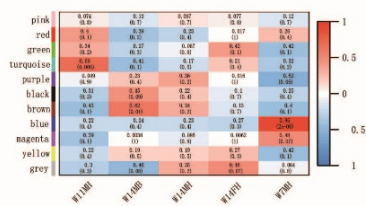

(E)

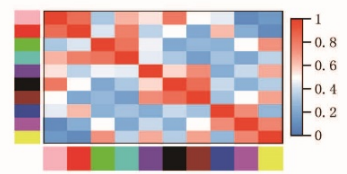

(B)

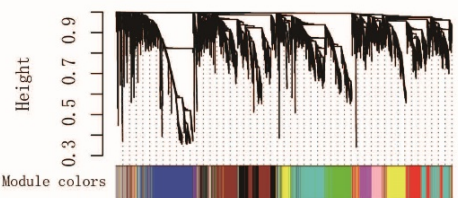

(D)
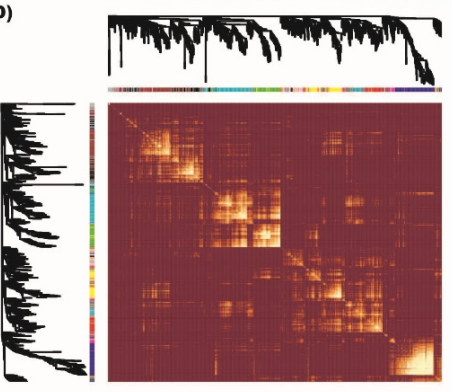

Figure 2. Genome-wide gene coexpression analysis of 15 RNA samples. (A) Cluster tree of 15 RNA samples. (B) In the cluster tree of genome-wide gene expression, genes with similar expression patterns belong to the same category. Each colored module represents a gene category. (C) Module-phenotype relationships. Each row and column represent a module and trait, respectively. The numbers in the boxes are the Pearson correlation coefficients of the modules and phenotypes. (D) Eigengene adjacency heat map. (E) Module-module relationships.
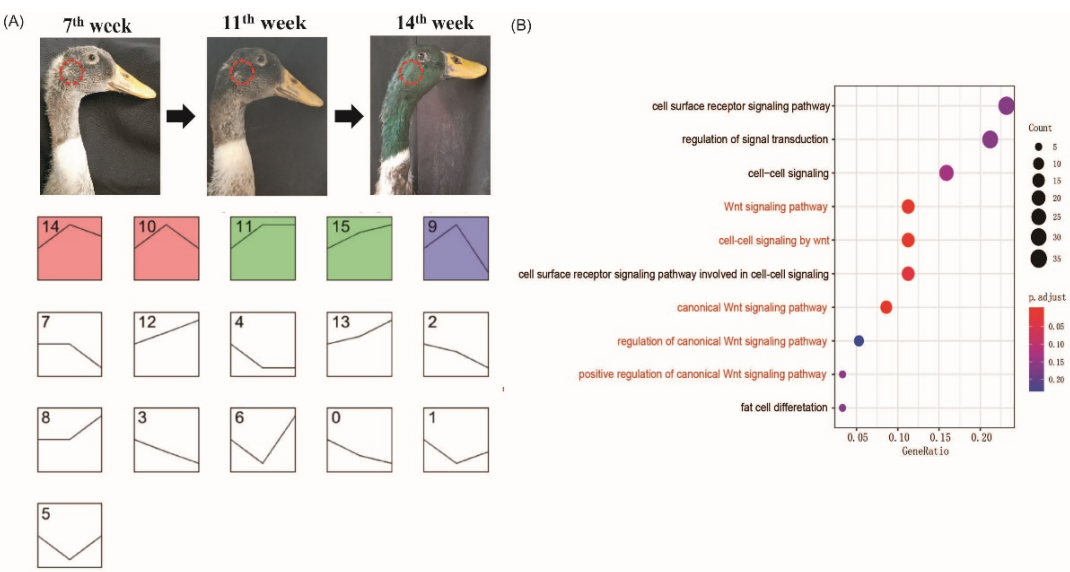

Figure 3. Genome-wide gene expression trend analysis. (A) Classification of genome-wide gene expression trends in three growth stages. Each simplified line chart shows a trend of gene expression. The vertical coordinates of the line graph are the FPKM value, and the horizontal coordinates are the growth 
stages. The sampling points on the mallards are indicated. (B) GO analysis of the genes in category 10.

(A)
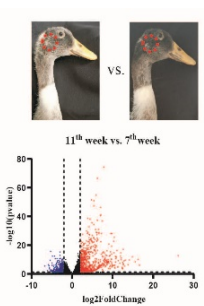
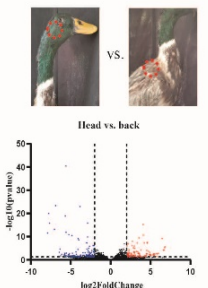
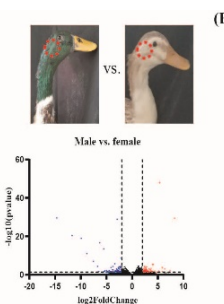

(B)

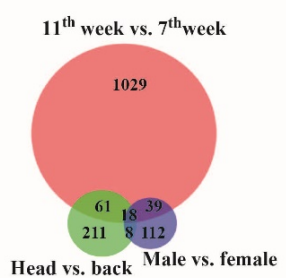

(C)
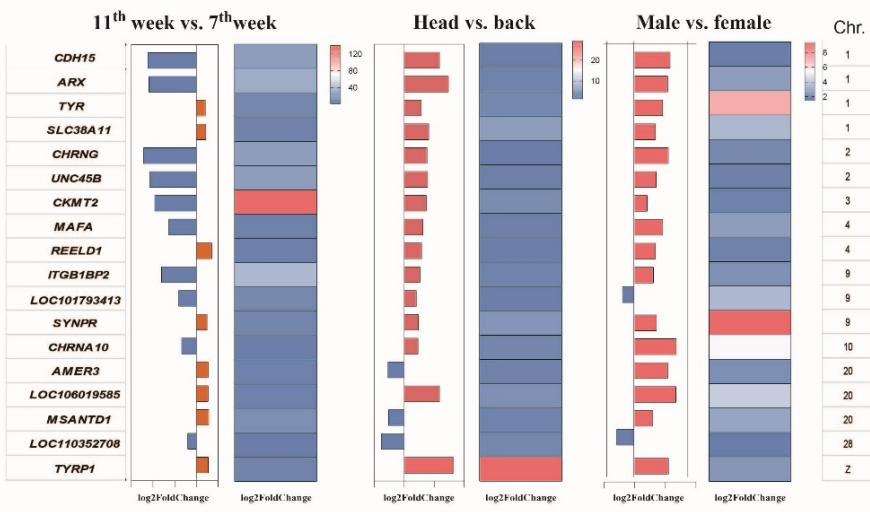

(D)

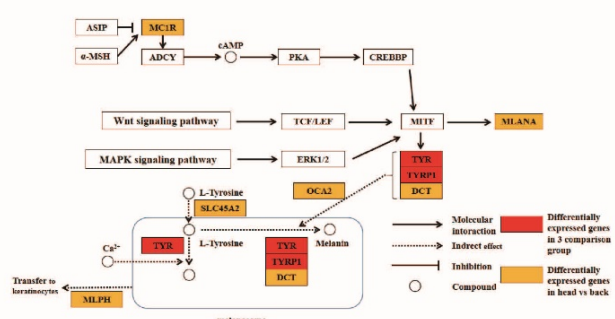

Figure 4. Screening of the causative genes of the male green head feathers. (A) Volcano plots showing the differentially expressed genes (DEGs) of 3 comparison pairs: $11 \mathrm{w}$ vs. $7 \mathrm{w}$, male vs. female, and head vs. back. We have indicated the sampling points in each image of a mallard. (B) The Venn diagram shows the number of DEGs among the three comparison groups. (C) The consensus genes that were differentially expressed among the three comparison groups. (D) The signaling pathway of melanin biosynthesis. 


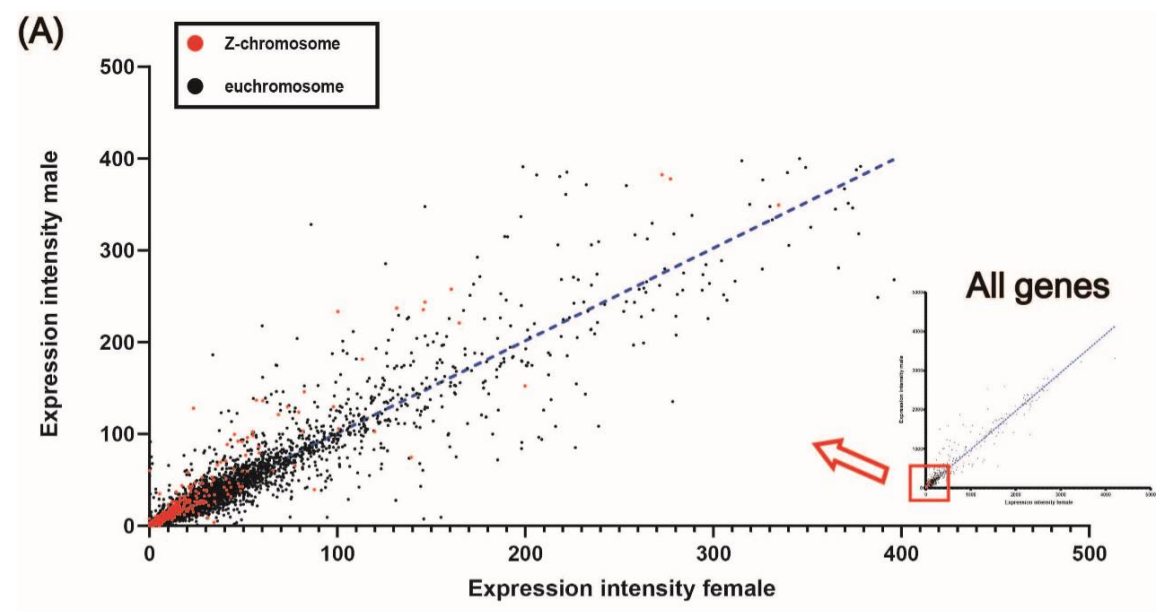

(B)

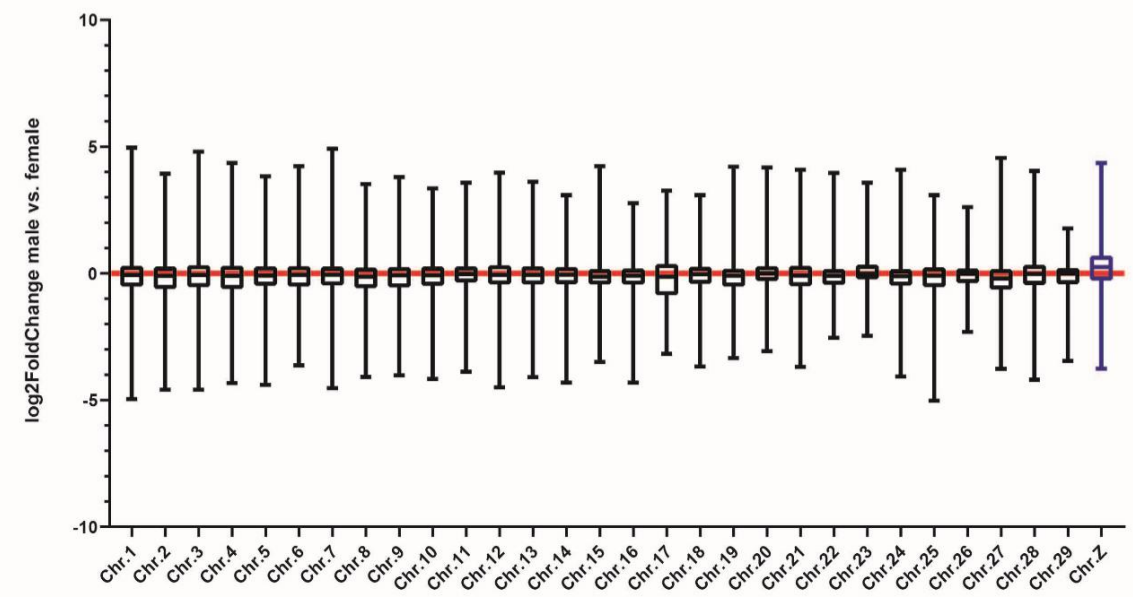

Figure 5. The expression patterns of Z- chromosome-linked genes in mallards. (A) The expression levels of individual Z-chromosome-linked and euchromosome-linked genes. Each point in the scatter diagram represents the FPKM value of a gene in the male and female head samples. (B) Box plots showing the median log2-fold change values per chromosome of male vs. female. The whiskers extend to the minimum and maximum values that are not outliers (outliers are defined by a log2 fold-change $>5$ or $<-5$ ). 

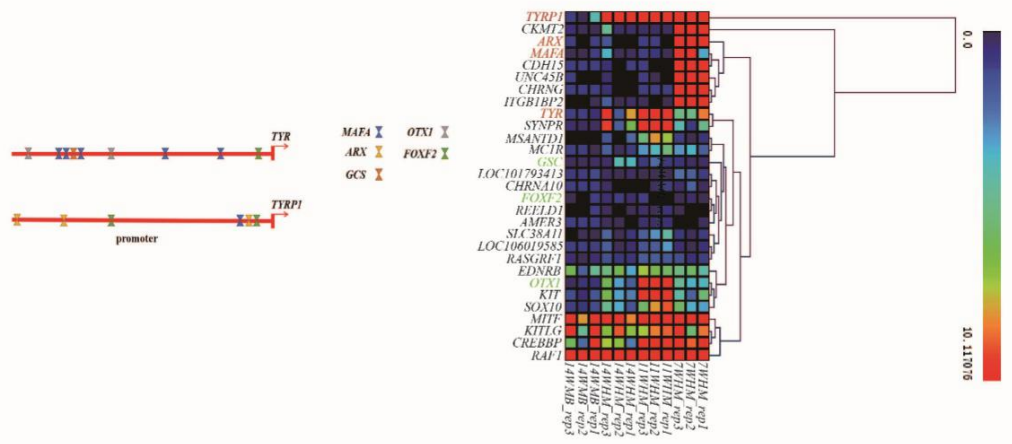

Figure 6. The effect of transcription factors on $T Y R$ and TYRP1 gene expression. (A) The TF binding sites of the TYR and TYRP1 promoters were predicted. (B) Expression patterns of some pigmentation genes and 18 causative genes in 12 male samples. We used the FPKM results for the above samples for heat map construction. 
(A)

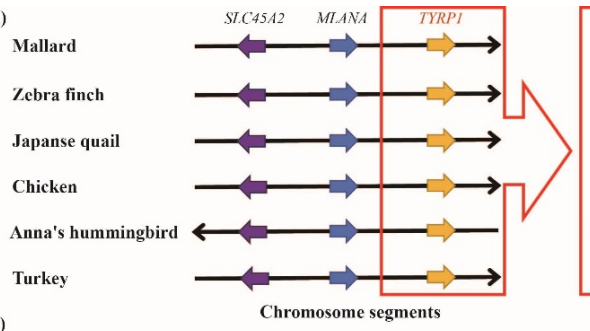

(B)

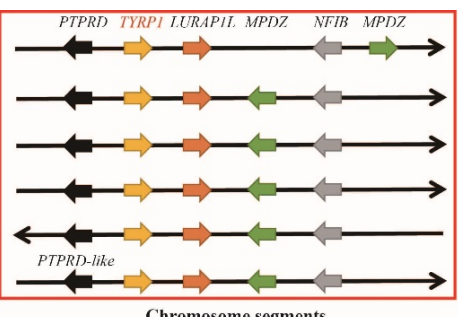

Chromosome segments

PTPRD

TYRPI

IURAPIL.

$M P D Z$

NFIB

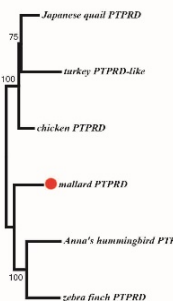

0.005

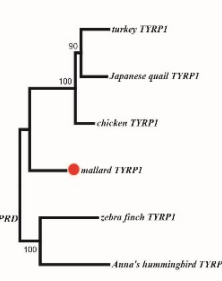

5.9
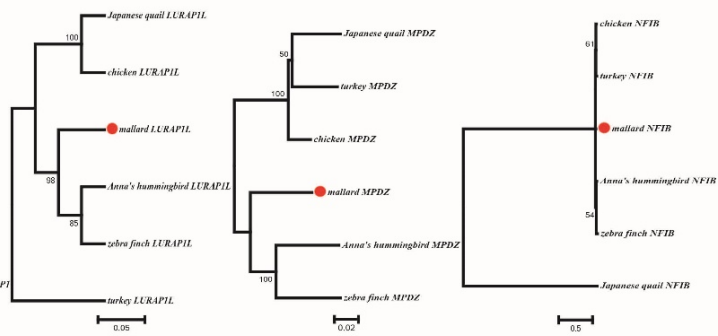

Figure 7. Synteny and phylogenetic analysis of TYRP1 and its neighboring genes in avians. (A) The relative positions of pigmentation genes and the neighboring genes of TYRP1 in Z-chromosome segments. Each arrow color represents a gene. We arranged the order of the arrows on the basis of the relative gene positions on the chromosome. (B) Phylogenetic trees of five neighboring genes of the TYRP1 gene. 

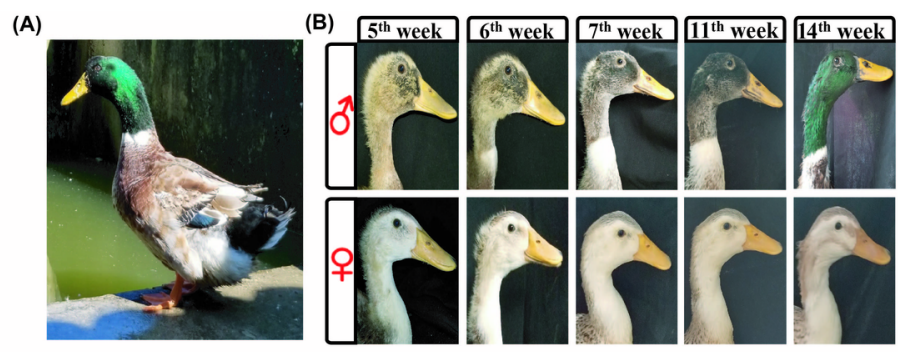

(C)

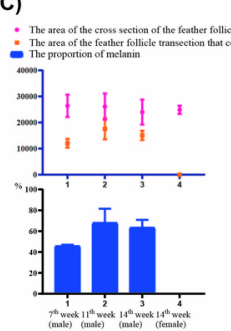

(D)

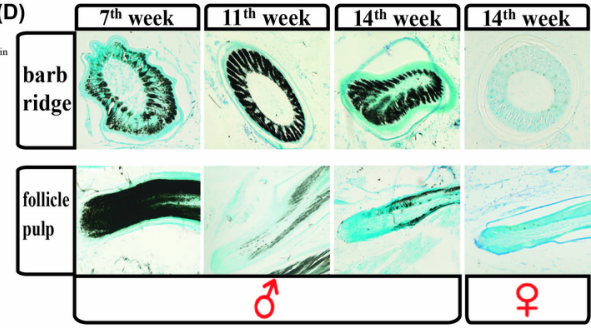

(E)

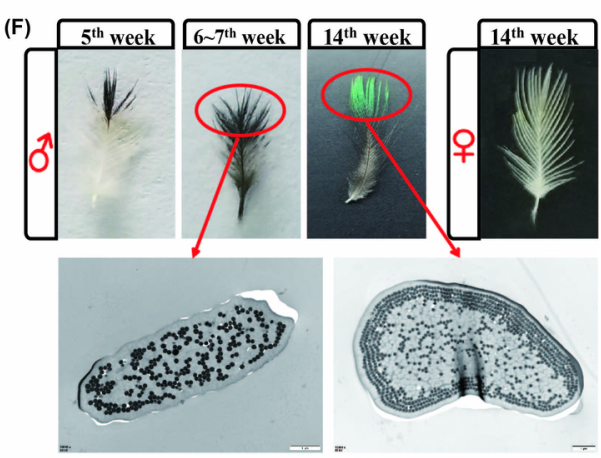


(A)

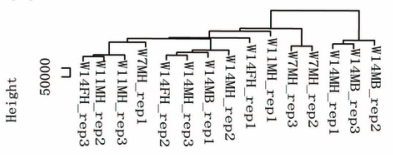

(C)

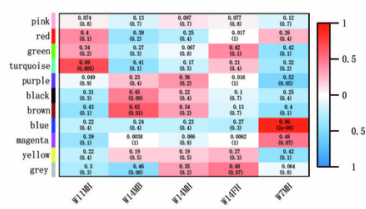

(E)

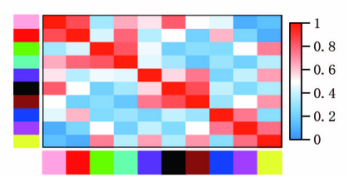

(B)

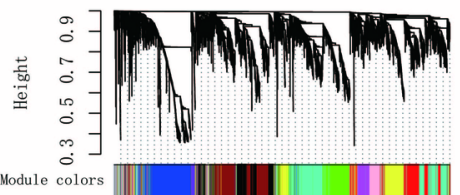

(D)
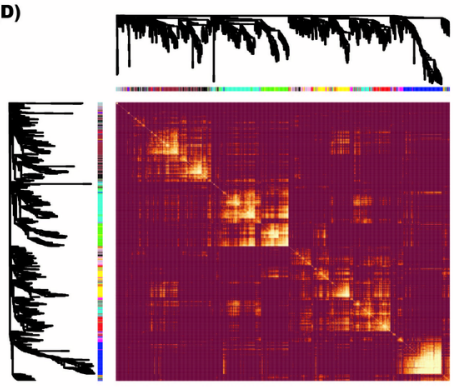

\section{Hosted file}

Figure_3.pdf available at https://authorea.com/users/324293/articles/452566-transcriptomeanalysis-reveals-that-tyrp1-linked-with-the-z-chromosome-causes-the-green-color-of-thefeathers-covering-the-head-of-male-mallards-through-sex-biased-expression 
(A)
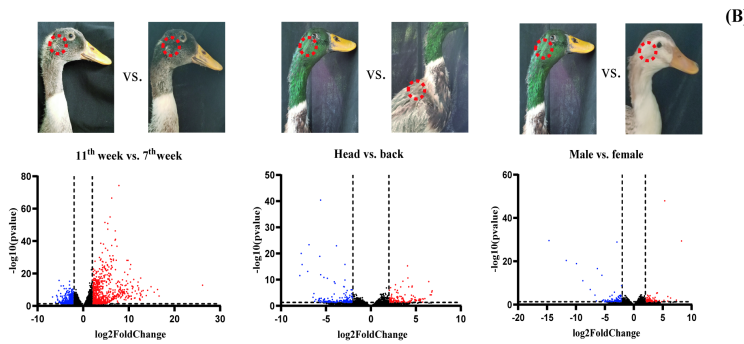

$11^{\text {th }}$ week vs. $7^{\text {th }}$ week

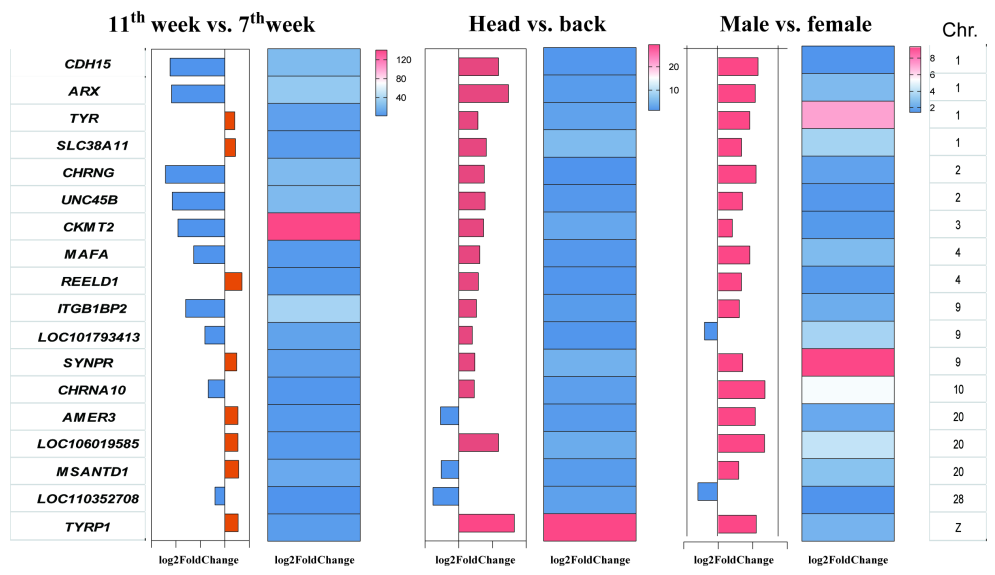

(D)

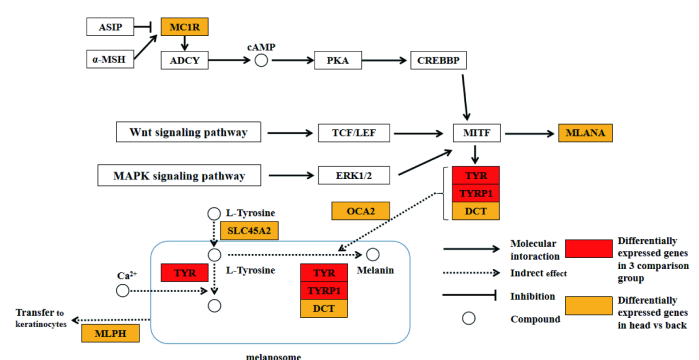




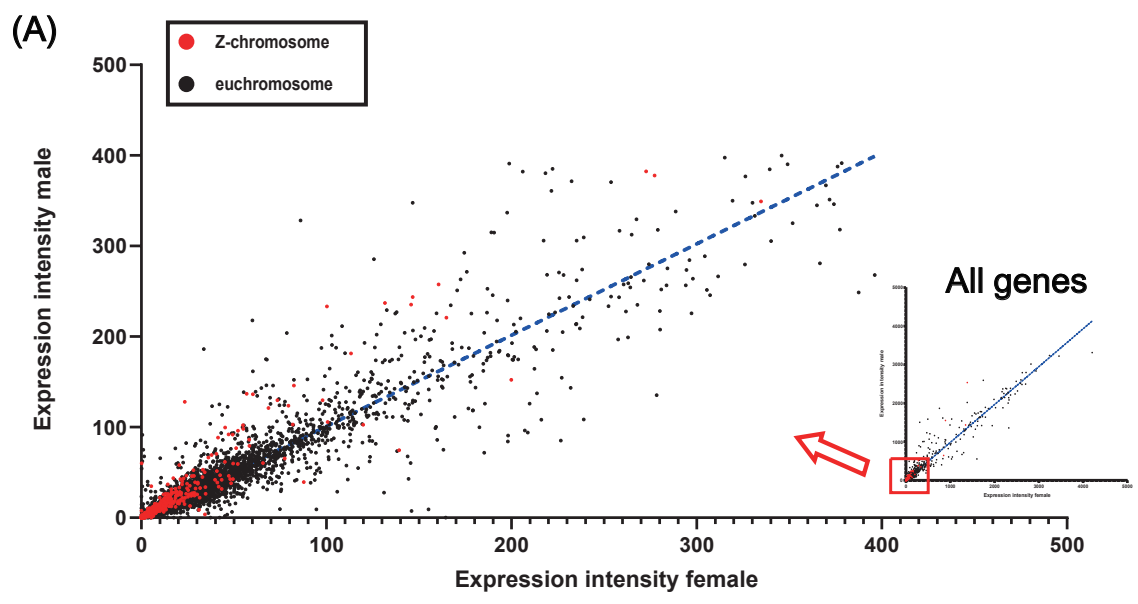

(B)

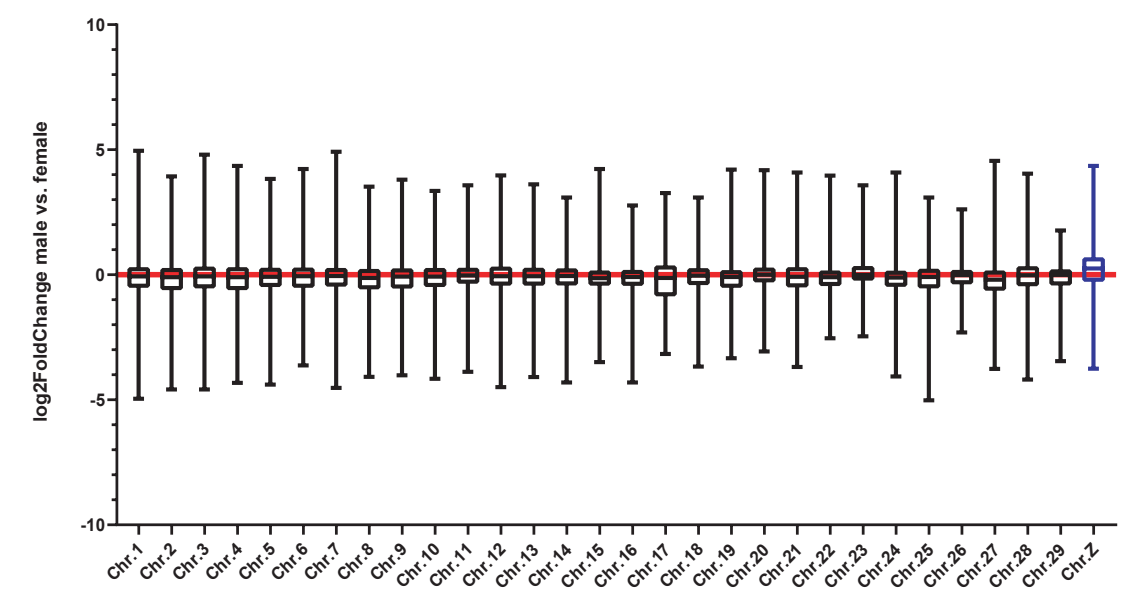


(A)

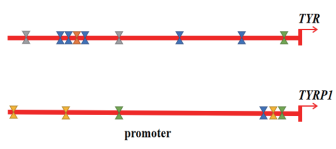

(B)

MAFA X OTXI
ARX Y

GCS I

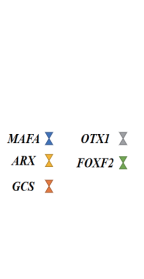

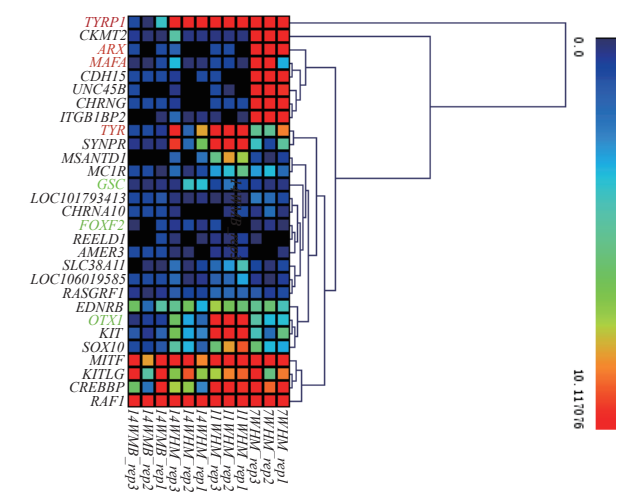

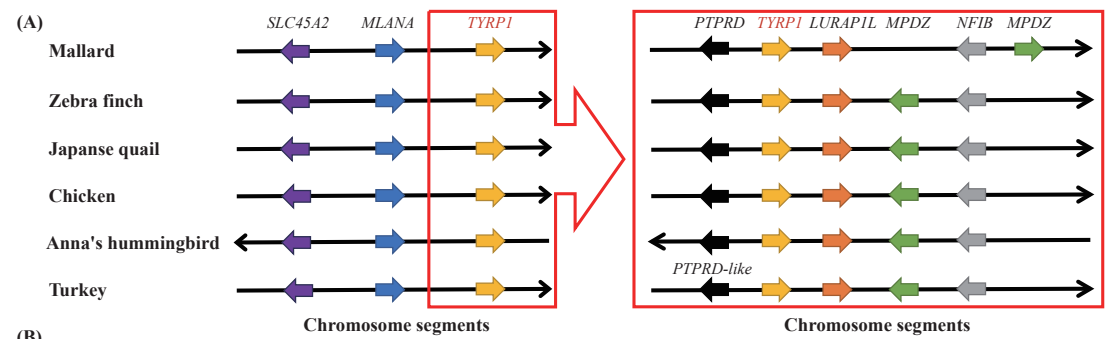

(B)
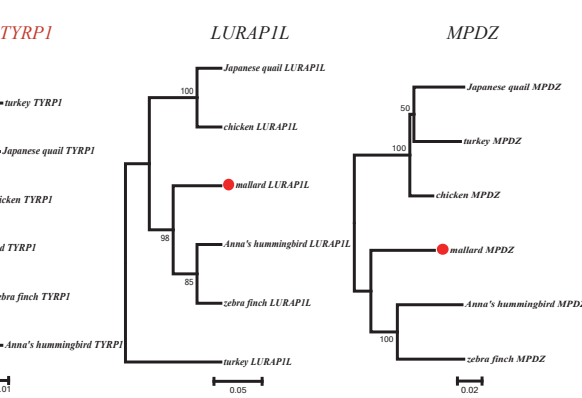

NFIB

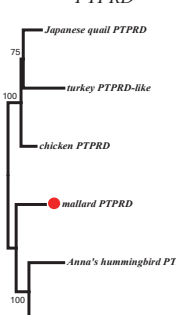

0.005

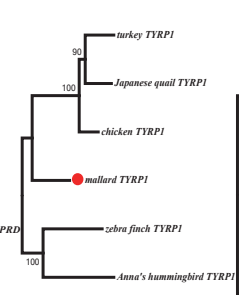

tr.
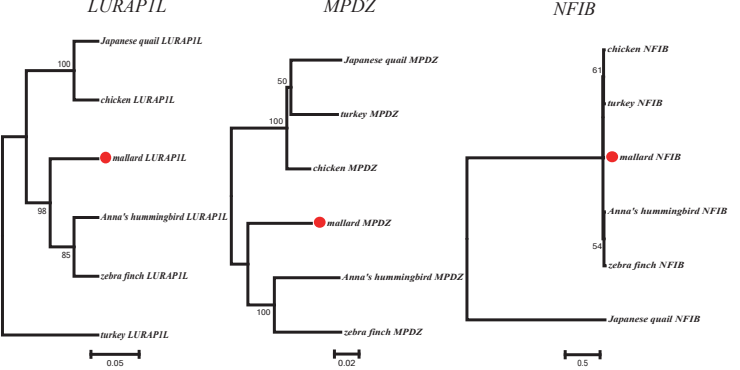